\title{
Application of acute whole-body vibration and lower-body exercise: effects on concentric torque in lower-limb muscles
}

\author{
Adam Hawkey ${ }^{1,2}$ Alexander Dallaway ${ }^{3}$ \\ ${ }^{1}$ School of Sport, Health and Social Sciences, Solent University, Southampton, UK; ${ }^{2}$ School of Medicine, Ninewells \\ Hospital and Medical School, University of Dundee, UK. ${ }^{3}$ Faculty of Health and Life Sciences, Coventry University, UK
}

\section{Summary}

Study aim: With contrary evidence regarding the effectiveness of acute whole-body vibration training (WBVT) on sporting performance, the current study examined WBVT's effect on concentric torque of the quadriceps (Q) and hamstrings (H).

Material and methods: Following ethical approval, 11 male team sport players (age: $22.9 \pm 3.3 \mathrm{yrs}$, height: $1.80 \pm 0.07 \mathrm{~m}$, mass: $82.5 \pm 12.6 \mathrm{~kg}$ ) completed three separate weekly WBVT sessions. Baseline and post - WBVT intervention measurements of $\mathrm{Q}$ and $\mathrm{H}$ concentric torque were recorded, using an isokinetic dynamometer, at each session. Isokinetic knee extension and flexion was performed at $180^{\circ} \mathrm{s}^{-1}$ through $90^{\circ}$ range of motion. For the training intervention, vibration amplitude remained at $2 \mathrm{~mm}$, while frequency was set at $0 \mathrm{~Hz}, 30 \mathrm{~Hz}$ or $50 \mathrm{~Hz}$; randomised so participants experienced one frequency per session. Torque data $(\mathrm{Nm})$ and $\mathrm{H}$ and $\mathrm{Q}$ ratio $(\mathrm{H}: \mathrm{Q})$ were analysed using 3-way and 2-way ANOVA with repeated measures respectively, with three within subjects' factors: frequency, muscle group and intervention.

Results: Main interaction effect (frequency x muscle group x intervention) was insignificant $(P=0.327)$. Significant muscle group $\mathrm{x}$ frequency $(P=0.029)$ and muscle group $\mathrm{x}$ intervention $(P=0.001)$ interactions were found. Intervention, regardless of WBVT, significantly increased concentric torque of $\mathrm{H}(P=0.003)$ and significantly reduced concentric torque of $\mathrm{Q}(P=0.031)$. While H: Q x frequency interaction was insignificant $(P=0.262)$, the intervention significantly improved H: Q $(P=0.001)$. Conclusions: Team sport athletes experience a muscle-specific response in peak concentric torque to lower-body exercise. Acute WBVT does not provide additional positive or negative effects on $\mathrm{Q}$ or $\mathrm{H}$ strength.

Key words: Vibration training - Isokinetic dynamometry - Hamstrings - Quadriceps

\section{Introduction}

Vibration can be described as a mechanical action characterised by periodic oscillatory motion about an equilibrium point [19]. Humans are exposed to vibrations on a daily basis in various waveforms, which can be delivered through a multitude of agents, including the use of a pneumatic drill [24], and during the operation of cars, boats and aircraft [32]. Exposure to the mechanical forces associated with vibration is generally viewed as deleterious to human health [38] and is tightly regulated in the workplace by the International Organization for Standardization (ISO) [22]. In contrast though, vibration exposure can also induce certain advantageous physiological responses, which have been exploited to improve neuromuscular performance, particularly in a sporting context $[5,6,10,12,18]$. Therefore, sports requiring high levels of power may benefit from incorporating whole-body vibration training (WBVT) into warm-up protocols [23].

Acute bouts of WBVT have been predominantly applied to athletic populations in order to transiently enhance neuromuscular performance; evidenced by power-lifters using WBVT to increase neural drive and maximise fasttwitch fibre recruitment prior to a lift [40] and by Olympic gymnasts utilising vibratory stimuli to facilitate improvements in jump performance, balance and flexibility [17]. The acute effects of WBVT have been attributed to potentiation of the stretch reflex through enhanced neural control [12]. Skeletal muscles eccentrically and concentrically contract rapidly in response to vertical vibrations [39] in an attempt to dampen the vibratory waves, which results in muscle length changes that are subsequently detected by the muscle spindles [4]. The primary sensory endings of the muscle spindles are excited by the muscle length changes, which cause a tonic contraction of the muscle 
[32]; a phenomenon first proposed by Hagbarth and Eklund [16] and known as the tonic vibration reflex (TVR).

It has been demonstrated that muscles subjected to vibration training have enhanced electromyographical (EMG) activity, above that of a maximal voluntary contraction (MVC) [35]. Even muscles in a fatigued state display higher mean power frequency during WBVT, which can be attributed to alterations in neuromuscular recruitment patterns [39]. However, the optimal biomechanical parameters of WBVT are currently undecided in the literature. Frequency level, essential in stimulating the muscles at their resonant frequency [5], varies greatly; while $60 \mathrm{~Hz}$ has been reported by some to induce the highest EMG activity in the quadriceps [27], others have suggested that a range of $30 \mathrm{~Hz}$ to $50 \mathrm{~Hz}$ is optimal to activate the same group of muscles [28].

With team sports placing a great demand on the lower limbs, such as the hamstrings $(\mathrm{H})$ and quadriceps $(\mathrm{Q})$ muscle groups, the ability of these muscles to produce force is vital for performance [13] and injury prevention [14]. Effective monitoring of the muscles ability to produce force, combined with a conventional strength ratio between the $\mathrm{H}$ and $\mathrm{Q}(\mathrm{H}: \mathrm{Q})$ enables the prediction of injuries, including anterior cruciate ligament (ACL) and hamstring strains, which are common in team sports [21]. The current study, therefore, was designed to investigate the effects of acute WBVT in combination with thigh-specific body-weight exercises on peak torque of the $\mathrm{H}$ and $\mathrm{Q}$ in male team sport players to establish the most effective frequency for neuromuscular improvements of key muscle groups in this population. It was hypothesised that WBVT will have a positive effect on peak concentric torque in the $\mathrm{Q}$ and $\mathrm{H}$ muscles.

\section{Materials and methods}

\section{Participants}

Following institutional ethical approval, and in accordance with the latest delineation of the Helsinki Declaration [46], 11 healthy male university team sport athletes (mean \pm standard deviation: height $=1.79 \pm 0.07 \mathrm{~m}$; mass $=82.5 \pm 12.6 \mathrm{~kg}$; age $=23 \pm 3 \mathrm{yrs}$.) were recruited via a convenience sampling method. Prior to testing, all participants gave written informed consent and completed a pre-activity readiness questionnaire (PAR-Q). All participants also confirmed verbally, throughout the study, that they were not suffering from any injuries or illnesses that would prevent or restrict their participation.

\section{Study design}

A randomised controlled design was chosen in order to negate any learning effects. Three testing sessions were completed in total, with each session separated by one week to allow sufficient recovery time between the respective resistance training/testing sessions [31]. Sessions were completed at the same time of day ( $\pm 1 \mathrm{hr}$.) to avoid the confounding influence of circadian variation [42]. Participants were instructed to refrain from the consumption of alcohol and undertaking any vigorous activity 24 hours prior to the testing sessions. Any data obtained was concealed from all participants until the end of the study to prevent competition bias.

\section{Performance testing protocol}

All sessions started with participants completing a generic 5-minute warm-up on a cycle ergometer (Monark 915E, Sweden) at 100W. Three successive sub-maximal knee extension and flexion exercises on an isokinetic dynamometer (Kin-Com, USA) at $180^{\circ} \mathrm{s}^{-1}$ were then performed; serving both as part of the warm-up, targeting specific muscle groups, and as familiarisation trials. During these submaximal tests, and for the subsequent baseline and re-testing, participants were seated in the isokinetic dynamometer with hips aligned $90^{\circ}$ to the trunk and with the seat belt securely fastened to anchor the participants to the chair; preventing unwanted assistance from the hips (Fig. 1).

A shin pad was placed around the participants' right ankle, superior to the medial malleolus, and a knee restrictor was used to prevent hip and knee movement (Fig. 1). Participants were instructed to place their hands across

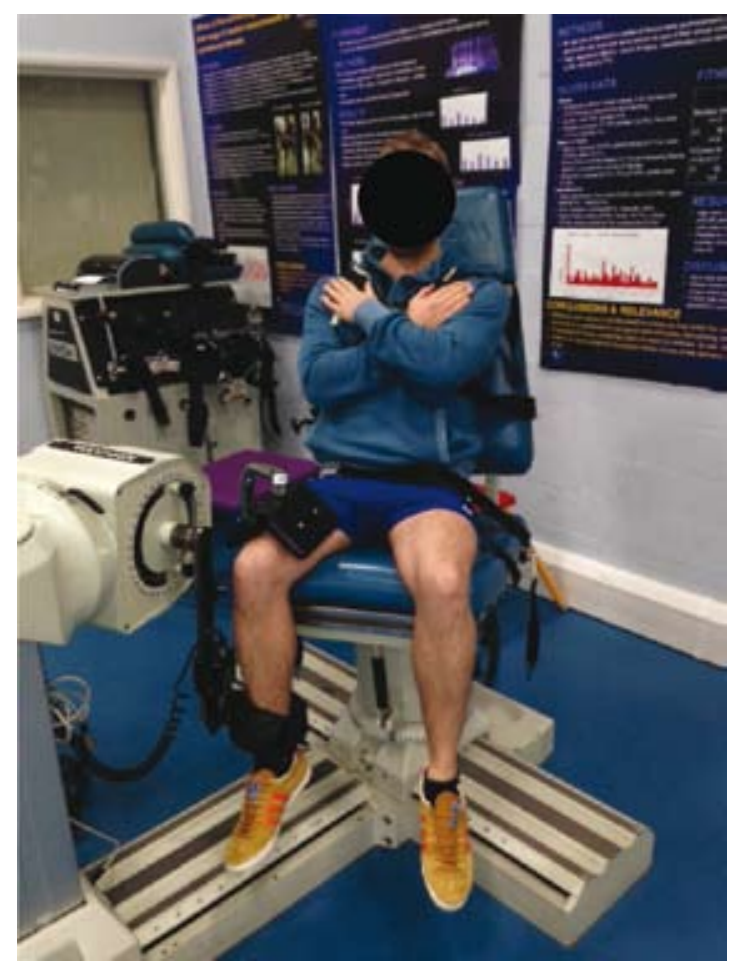

Fig. 1. Positioning of participant on the isokinetic dynamometer 


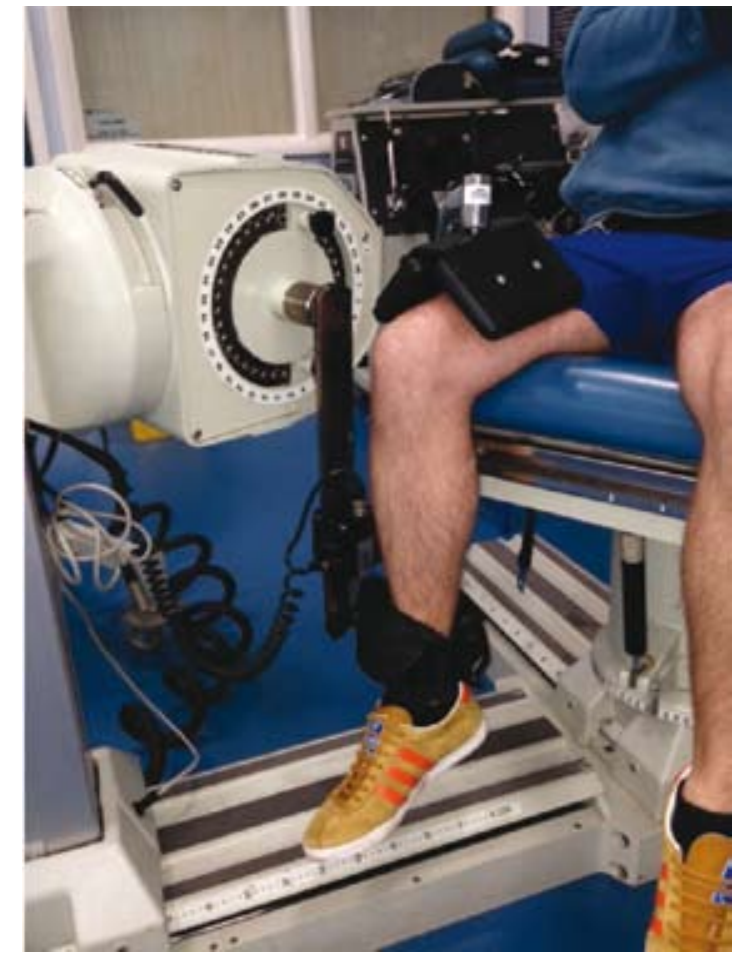

Fig. 2. Alignment of knee joint with the lever arm

their chest throughout the testing. The seat and level arm were manually adjusted to the specific requirements of each participant, with the axis of the lever arm visually

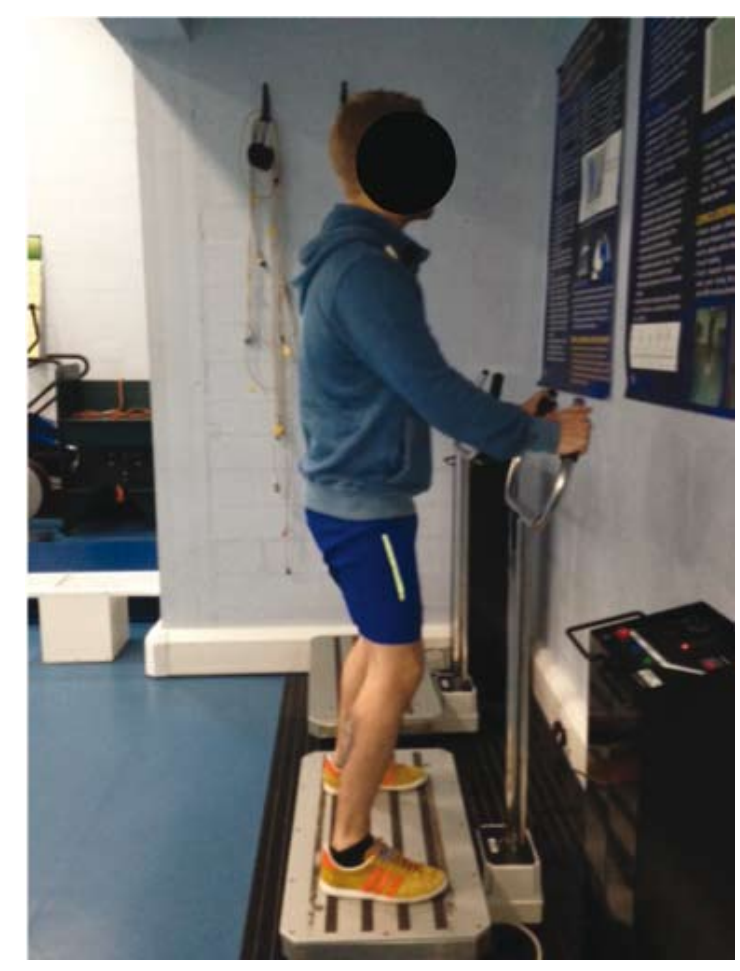

Fig. 3. Position 1: Standing (knee flexion $25^{\circ}$ ) aligned with the lateral femoral epicondyle of the participants' knee joint (Fig. 2). For the baseline tests participants flexed their knee to $90^{\circ}$ then performed three maximal successive contractions of the $\mathrm{Q}$ and $\mathrm{H}$ muscles through a range of motion (ROM) of $90^{\circ}$. The angular velocity of the dynamometer was set to $180^{\circ} \mathrm{S}^{-1}$. Peak torque was recorded for the $\mathrm{H}$ and $\mathrm{Q}$ for each trial and the $\mathrm{H}: \mathrm{Q}$ was calculated by dividing the peak torque of the $\mathrm{H}$ by the peak torque of the $\mathrm{Q}$.

\section{Intervention protocol}

Immediately following the baseline tests, all participants undertook a $60 \mathrm{~s}$ passive recovery before completing the WBVT intervention. A NEMES Bosco vibration platform (NEMES Bosco, Greece) was used to administer the WBVT stimulus. Amplitude was maintained at $2 \mathrm{~mm}$ throughout the three different frequency conditions $(0 \mathrm{~Hz}$, $30 \mathrm{~Hz}, 50 \mathrm{~Hz}$ ). All participants experienced each of these three conditions, one per week, in a randomised order. Five positions/exercises were performed during the vibration intervention: Standing posture with knees flexed at $25^{\circ}$ (Fig. 3); isometric squat with knees flexed $80^{\circ}$ (Fig. 4); dynamic squat (knee flexion from $25^{\circ}$ to $80^{\circ}$ to $25^{\circ}$ ) at a tempo of 2 seconds up 2 seconds down (Fig. 5); isometric right (Fig. 6) and left leg lunge with the front knee flexed to $90^{\circ}$. These positions/exercises were based on a previous study by Cochrane and Stannard [6] and chosen as they are commonly performed on WBV platforms and wellsuited to WBVT.

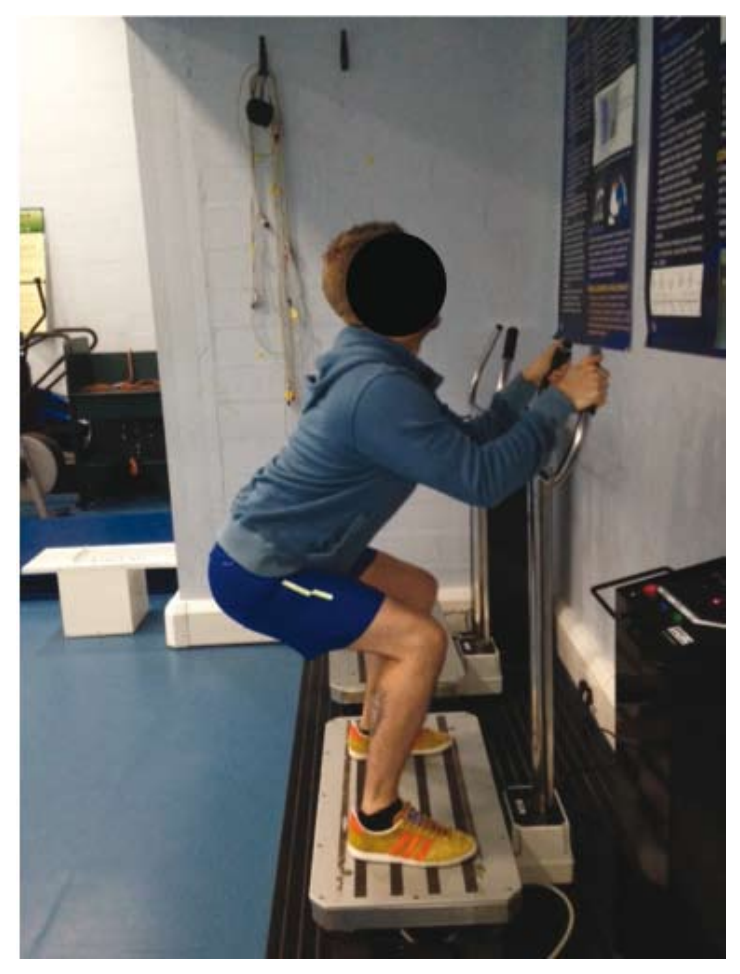

Fig. 4. Position 2: Squat (knee flexion $80^{\circ}$ ) 

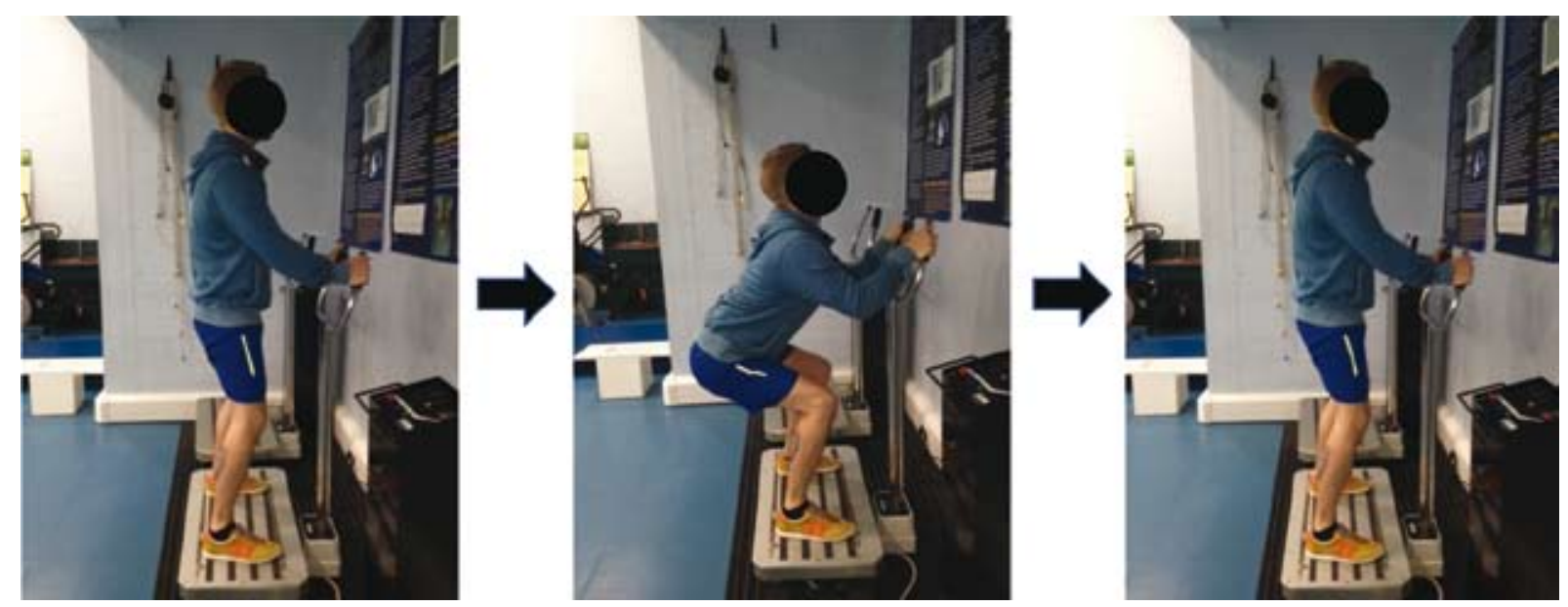

Fig. 5. Position 3: Dynamic squat (knee flexion from $25^{\circ}$ to $80^{\circ}$ to $25^{\circ}$ )

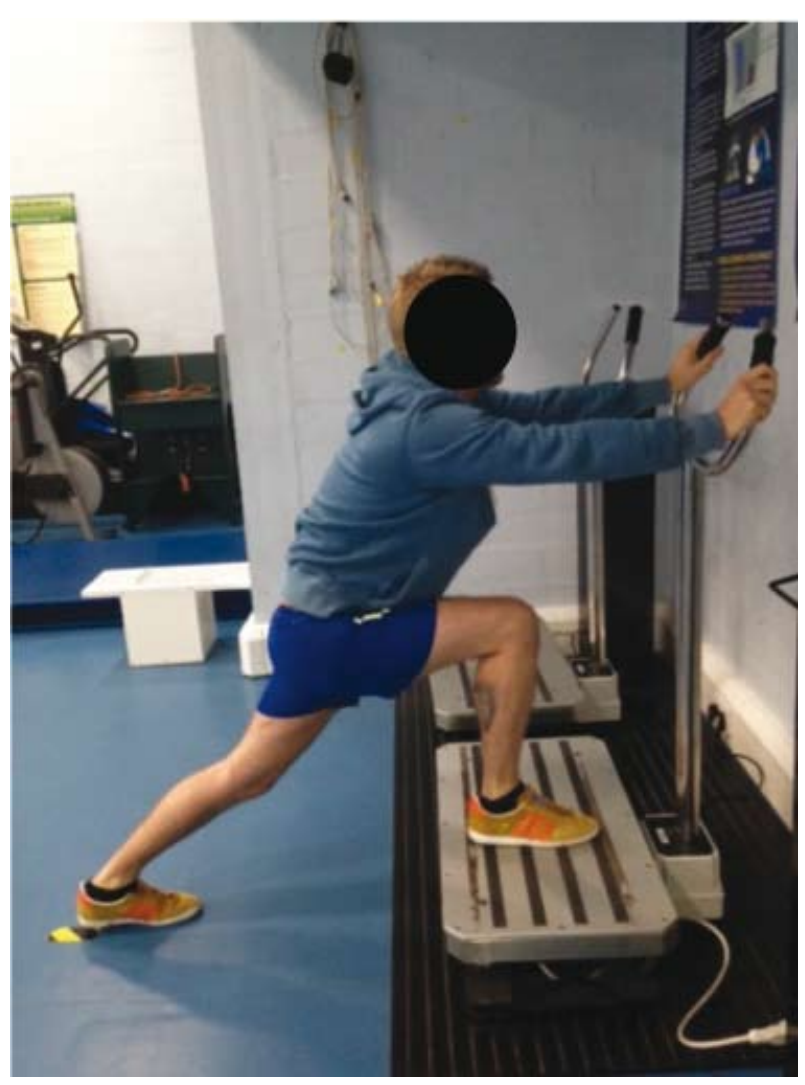

Fig. 6. Position 4 [and 5]: Right leg lunge with front knee flexion at $80^{\circ}$ (this was repeated with the left leg [not shown])

Participants were required to maintain their centre of mass centrally so that additional loading was not placed on either leg. The first three positions were performed for $60 \mathrm{~s}$ each and the lunges for $30 \mathrm{~s}$ on each leg. During the standing and squatting exercises, participants stood on the platform with feet shoulder width apart and knees flexed to prevent transmission of vibratory forces to the head [38]. Footwear was also controlled with participants required to wear the same footwear for every session to standardise vibration dampening. As acute WBVT can impair proprioception [9], as the muscle spindles are desensitised immediately following exposure [23], and in accordance with American College of Sports Medicine (ACSM) recommendations regarding resistance-type training [37], a delay of $60 \mathrm{~s}$ between each vibration exposure was incorporated into the protocol. Following completion of the WBVT intervention a $60 \mathrm{~s}$ passive recovery was given before participants were re-tested on the isokinetic dynamometer using the same procedure as for the baseline tests. A cool-down consisting of 4-min on a cycle ergometer at $100 \mathrm{~W}$ was performed upon completion of the post-intervention testing. The entire performance testing protocol can be seen in Figure 7.

\section{Statistical analysis}

The torque data $(\mathrm{Nm})$ and ratio data $(\mathrm{H}: \mathrm{Q})$ were analysed using a 3-way and 2-way ANOVA with repeated measures, respectively (SPSS, v20). All factors were within subjects. For the torque data, the factors were frequency (3 levels: $0 \mathrm{~Hz}, 30 \mathrm{~Hz}, 50 \mathrm{~Hz}$ ), muscle group (H vs. Q) and intervention (baseline vs. post intervention). For H: Q the factors were frequency ( 3 levels: $0 \mathrm{~Hz}, 30 \mathrm{~Hz}, 50 \mathrm{~Hz}$ ) and intervention (baseline vs. post intervention). Followup paired-samples T-tests were performed to assess the change in peak torque following the intervention conditions. Cohen's d effect size estimates were calculated for pre-post comparisons. Significance was assumed at a $95 \%$ level of confidence.

\section{Results}

The main interaction effect (vibration frequency $\mathrm{x}$ muscle group $\mathrm{x}$ intervention) was not significant $(P=0.327)$. ANOVA revealed significant muscle group $\times$ vibration 


\begin{tabular}{|c|c|c|c|c|c|c|}
\hline WARM-UP & BASELINE TEST & RECOVERY & WBVT INTERVENTION & RECOVERY & POST-INTERVENTION TEST & COOL DOWN \\
\hline $\begin{array}{l}\text { Cycle } \\
\text { ergometer } \\
\text { at } 100 \text { Watts }\end{array}$ & $\begin{array}{l}3 x \text { successive submaximal } \\
\text { voluntary contractions of the } \\
\text { quadriceps and hamstrings } \\
3 x \text { successive maximal } \\
\text { voluntary contractions of the } \\
\text { quadriceps and hamstrings }\end{array}$ & $\begin{array}{l}\text { Passive } \\
\text { recovery } \\
\text { following } \\
\text { baseline tests }\end{array}$ & 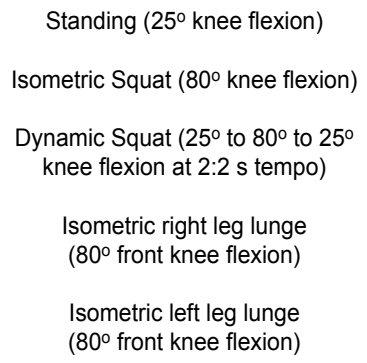 & $\begin{array}{l}\text { Passive } \\
\text { recovery } \\
\text { following } \\
\text { WBVT } \\
\text { intervention }\end{array}$ & $\begin{array}{l}\text { 3x successive maximal } \\
\text { contractions of the quadriceps } \\
\text { and hamstrings }\end{array}$ & $\begin{array}{l}\text { Cycle } \\
\text { ergometer } \\
\text { at } 100 \text { Watts }\end{array}$ \\
\hline $5 \mathrm{~min}$ & & $60 \mathrm{~s}$ & $\begin{array}{l}60 \mathrm{~s} \text { for each of positions } 1-3, \\
30 \mathrm{~s} \text { for each of positions } 4-5, \\
60 \text { s recovery between positions }\end{array}$ & $60 \mathrm{~s}$ & & $4 \min$ \\
\hline
\end{tabular}

Fig. 7. Warm-up, baseline, WBVT intervention and post-intervention test protocol

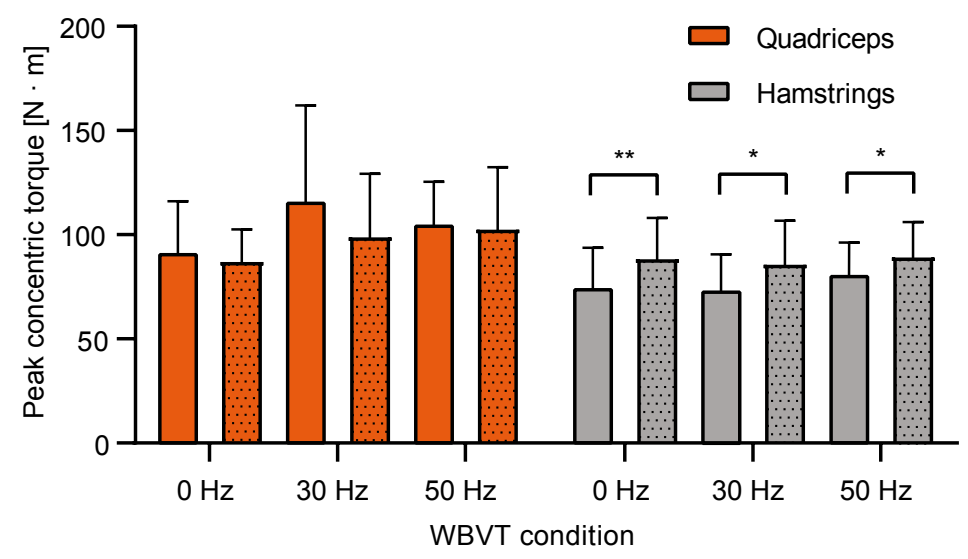

Fig. 8. Pre- and post-peak torque values (mean \pm SD) for the quadriceps and hamstrings for each acute training intervention condition. Solid bars represent baseline measurements, dotted bars represent post intervention measurements. ${ }^{* *}-\mathrm{p}<0.01$, $*-\mathrm{p}<0.05$

frequency $(P=0.029)$ and muscle group $\times$ intervention $(P=0.001)$ interaction effects. There was no significant $\mathrm{H}: \mathrm{Q} \times$ vibration frequency interaction effect $(P=0.262)$, however, a significant $\mathrm{H}: \mathrm{Q} \times$ intervention interaction was observed $(P=0.001)$ (Fig. 8$)$.

The quadriceps and hamstrings concentric torque was not significantly affected by the two vibration frequencies $(30 \mathrm{~Hz}$ and $50 \mathrm{~Hz}$ ) compared to the sham $(0 \mathrm{~Hz})$ condition. However, the exercise protocol induced significant changes in both muscle groups. ANOVA also revealed the quadriceps concentric torque significantly decreased following the intervention regardless of WBVT exposure $(P=0.031)$. However, $\mathrm{H}$ concentric torque was significantly increased following the intervention regardless of WBVT $(P=0.003)$. Post-hoc tests revealed significant increases in $\mathrm{H}$ concentric torque following the intervention for the $0 \mathrm{~Hz}(P=0.007), 30 \mathrm{~Hz}(P=0.083)$ and $50 \mathrm{~Hz}$ $(P=0.016)$ conditions. No significant differences were found for $\mathrm{Q}$ peak torque following any of the intervention conditions. Effect size estimates (Cohen's d) for the intervention on $\mathrm{Q}$ concentric torque were 0.22 for the sham condition, 0.44 for $30 \mathrm{~Hz}$ WBVT and 0.10 for $50 \mathrm{~Hz}$ WBVT. The effect of acute exercise and WBVT was greater on concentric torque in the H. For the sham condition, a large effect size was calculated at 0.73 . Moderate to large effect size were also found for the $30 \mathrm{~Hz}$ and $50 \mathrm{~Hz}$ conditions, 0.66 and 0.53 , respectively.

\section{Discussion}

The current study examined how acute WBVT at different frequencies affects concentric torque of the quadriceps and hamstrings. The main finding was vibration frequencies did not have a significant effect on the two muscle groups. However, and contrary to expectations, the protocol used, regardless of exposure to WBVT, was able to significantly increase the concentric torque of the hamstrings while significantly reducing the concentric torque of the quadriceps. 


\section{WBVT effects on the quadriceps}

The quadriceps responded in a similar way to all of the vibratory conditions. Exposure to vibration at $30 \mathrm{~Hz}$ and $50 \mathrm{~Hz}$ induced the same acute adaptations from the quadriceps as the sham $(0 \mathrm{~Hz})$ condition. These findings are substantiated by another study, which found that an acute bout of WBVT induced the same adaptations in the knee extensor muscles as an identical protocol without vibration [7]. Exposure to vibration has been suggested to lower recruitment thresholds of fast-twitch motor units [39], which are able to elicit improvements in quadriceps torque at high speeds; as fast-twitch muscle fibres are recruited for high force contractions [30]. However, the sub-elite population used in the current study may have experienced fatigue due to not possessing sufficient musculature to dampen the vibrations. In contrast, according to Mester et al. [32], the stiff muscle-tendon complex of elite athletes is well developed and therefore more likely able to dampen such vibrations.

The performance test used may also explain why no changes were observed for the vibration conditions. Vibration has been shown to potentiate the stretch reflex [12]; therefore, dynamic movements incorporating a stretch shortening cycle (SSC), such as a vertical counter-movement jump, may elicit greater improvements than the MVCs used in the current study [23]. This is supported by research from Cormie et al. [10] who found that while strength did not respond to acute WBVT, vertical jump performance improved. The isokinetic speed used $\left(180^{\circ} \mathrm{s}^{-1}\right)$ may also explain why no changes were found in quadriceps torque post vibration. Knee extension torque has improved following a similar vibration protocol when an isokinetic speed of $60^{\circ} \mathrm{s}^{-1}$ has been used [40]. Slower isokinetic speeds would allow the muscle to develop maximal tension at the optimal joint angle [3] rather than the angular velocity being too fast reflecting the muscle's inability to develop maximum tension at the optimal joint angle [11].

\section{WBVT effects on the hamstrings}

WBVT at the two frequencies $(30 \mathrm{~Hz}$ and $50 \mathrm{~Hz})$ had a similar effect on the concentric torque of the hamstrings as the sham vibration $(0 \mathrm{~Hz})$ condition. This resulted in an insignificant interaction effect between vibration frequencies and hamstring torque. These findings are supported by Siu et al. [41] who also found the concentric torque of the hamstrings did not significantly change post-vibration but are contrary to the findings of Avelar et al. [2] who suggest that the concentric torque of the knee flexors significantly increases after an acute bout of WBVT. While Avelar et al. [2] used a similar population and vibration magnitude to the current study the isokinetic velocity at which knee flexion was performed was significantly lower, with peak concentric torque measured at $60^{\circ} \mathrm{s}^{-1}$, compared to $180^{\circ} \mathrm{s}^{-1}$ for the current study. Therefore, the slow and fast twitch muscle fibres in the hamstrings may respond through different mechanisms to WBVT resulting in an enhanced force output at slower angular velocities.

\section{Detrimental effects of the exercise protocol on the quadriceps}

The exercises performed significantly reduced the concentric torque in the quadriceps regardless of exposure to WBVT. As both squatting and lunging exercises are closed kinetic chain movements, involving hip and knee extensor muscle groups and ankle plantar flexors, and activating the vastus lateralis and vastus medialis muscles respectively $[29,36]$, these positions likely placed an increased gravitational load on the dynamic stabilising structures. The quadriceps have been shown to experience greater forces at greater angles of knee flexion and as participants spent the majority of the time performing the intervention with large degrees of knee flexion, the quadriceps would have been subjected to high forces [33]; linking to previous reports of greater activation in the quadriceps with increasing knee flexion [15]. It is reasonable to assume that this increased demand caused fatigue [44] as the population likely did not possess adequate musculature in the knee extensor muscles to attenuate fatigue caused by the intervention exercises; further explaining the decrement in the quadriceps concentric torque. This can be further supported by elite team sports populations exhibiting improvements in lower-limb force output following squatting exercises [26]; although it is important to note here that, in this particular example, improvements were only seen eight minutes after the squatting exercises had been performed. As the participants in the current study rested for only one minute following the intervention, the brief rest period may also help to explain why a reduction in the quadriceps torque was observed.

When performing maximal isokinetic knee extensions, the concentric contraction of the quadriceps causes a co-contraction of the hamstrings especially towards full knee extension [1]. Co-contraction of the hamstrings has also shown to increase with increasing fatigue of the quadriceps during isokinetic knee extensions [43]. As the quadriceps were fatigued from the exercises performed, the greater co-contraction of the hamstrings would have contributed to reducing the peak torque in the quadriceps during the isokinetic knee extensions by resisting the agonists' concentric contraction [25]. As co-contraction of the hamstrings limits the force production of the quadriceps during concentric contractions the action of the hamstrings may have limited the torque in the quadriceps. 


\section{Ergogenic effects of the exercise protocol on the hamstrings}

The exercises performed significantly increased the concentric torque of the hamstrings irrespective of exposure to WBVT. It is likely that as the quadriceps fatigued the hamstrings would have been activated to a greater degree to resist forces about the knee joint; as the hamstrings function to support the ACL in resisting antero-posterior forces [1]. Squatting exercises have been shown to activate the hamstrings significantly less than other hamstring exercises [47] and the squat position adopted for much of the exercise intervention during the current study may have prevented excessive loads being applied to the hamstrings; the low demand therefore likely insufficient to induce fatigue. Furthermore, when the participants performed the isokinetic knee flexions, the resistive force of the knee extensors was diminished due to their fatigued state and potential reduction in neural drive. Eccentric exercises reduce the neural drive to agonist muscles [45]; as the quadriceps eccentrically contracted during the interventions, neural drive to the quadriceps may have been reduced. This enabled the hamstrings to produce greater concentric torque than for the preintervention measurements as the quadriceps muscles were not able to co-contract to the same degree and resist the agonists' concentric contraction. In a fatigued state, concentric contractions of the quadriceps have shown to result in a significant increase in hamstring co-activation [48]. With the quadriceps likely fatigued due to the exercises, the hamstrings would have received a potentiating stimulus from the exercises without having to provide dynamic stability. As the participants performed the squats and lunges with upright trunks the hamstrings were not recruited to a great degree, potentially preventing fatigue [34].

\section{Effects of WBVT and the exercise protocol on the $H: Q$}

Results suggest WBVT did not directly affect the H: Q although the exercises performed significantly improved the H: Q. The exercises performed, regardless of WBVT exposure, were able to significantly improve the $\mathrm{H}$ : $\mathrm{Q}$ to values of approximately 1.0 , which is the ratio value indicating the equality of the hamstrings to provide counteracting flexor moments against the extensor moments exerted by the quadriceps [8]; specifically recommended for the prevention of ACL injuries [20]. This was to be expected as the hamstrings significantly improved and the quadriceps significantly decreased following the exercise intervention, regardless of WBVT.

\section{Limitations}

Whilst the current study employed a rigorous methodology, there were limitations that should be acknowledged. Primarily, there was no control condition in which participants would have performed no exercises and no exposure to WBVT. This may have enabled us to separate the effects of the exercise protocol from the WBVT further. Also, isokinetic testing was performed at $180^{\circ} \mathrm{s}^{-1}$ only. A high angular velocity was selected as one of the most widely accepted mechanisms of WBVT in augmenting strength output is through the lowering of fast-twitch motor unit recruit thresholds. However, using a range of angular velocities may have provided a more comprehensive evaluation of the acute effects of WBVT in relation to muscle function characteristics of the lower limb muscles. Other studies have shown improvements in knee extension torque at slower angular velocities after WBVT. Therefore, future research should look to incorporate a greater range of isokinetic testing conditions to further understanding on the effects of WBVT on muscular strength in the lower limbs.

Finally, the intervention exercises primarily targeted the quadriceps muscles which may have caused the apparent decline in knee extension torque. Whilst these exercises involve co-contraction of the hamstrings, exercises specifically targeting the hamstrings may have yielded different results. A range of exercises should be evaluated in future studies to investigate the effects of targeted exercise protocols on lower-limb strength.

\section{Conclusion}

The current study shows that acute WBVT at $30 \mathrm{~Hz}$ and $50 \mathrm{~Hz}$ does not affect the H: Q or peak concentric torque of the hamstrings or quadriceps at high speeds $\left(180^{\circ} \mathrm{s}^{-1}\right)$ and therefore suggests that WBVT may not be an effective training modality for transient short-term improvements in lower-limb force output in team sport athletes. The acute effects of WBVT on neuromuscular performance therefore remain equivocal and clarity regarding the biomechanical parameters, which are optimal to induce the TVR, is still required. However, the exercises performed in the current study augmented the hamstrings and deteriorated the quadriceps regardless of exposure to WBVT. The H: Q was also significantly improved by the exercises performed irrespective of WBVT, indicating that there is potential for the exercise protocol to be used in team sport warm-ups to reduce lower-limb injury risk. Future research should investigate the efficacy of WBVT as a training modality to ascertain whether chronic exposure elicits neuromuscular adaptations, which could improve lower-limb force output. Furthermore, the literature suggests elite populations benefit from acute WBVT more than recreational populations as elite athletes' muscle fibres and central nervous system have a higher sensitivity in response to additional stimulation. Therefore, the WBVT magnitudes used in the current study should be applied to a more elite population to discern whether level of competition affects how athletes respond to WBVT. 
Conflict of interest: Authors state no conflict of interest.

\section{References}

1. Aagaard P., Simonsen E.B., Andersen J.L., Magnusson S.P., Bojsen-Møller F., Dyhre-Poulsen P. (2000) Antagonist muscle coactivation during isokinetic knee extension. Scand. J. Med. Sci. Sports, 10(2): 58-67. DOI: 10.1034/j.1600-0838.2000.010002058.x.

2. Avelar N.C., Salvador F.S., Ribeiro V.G.C., Vianna D.M.S., Costa S.J., Gripp F., Coimbra C.C., Lacerda A.C.R. (2014) Whole body vibration and postactivation potentiation: a study with repeated measures. Int. J. Sports Med., 35: 651-657. DOI: 10.1055/s-00331354360.

3. Baltzopoulos V., Brodie D.A. (1989) Isokinetic dynamometry: applications and limitations. Sports Med., $8(2)$ : 101-116. DOI: 10.2165/00007256-19890802000003.

4. Cardinale M., Bosco C. (2003) The use of vibration as an exercise intervention. Exerc. Sport Sci. Rev., 31(1): 3-7. DOI: 10.1097/00003677-200301000-00002.

5. Cardinale M., Wakeling J. (2005) Whole body vibration exercise: are vibrations good for you? Br. J. Sports Med., 39(9): 585-589. DOI: 10.1136/bjsm.2005.016857.

6. Cochrane D.J., Stannard S.R. (2005) Acute whole body vibration training increases vertical jump and flexibility performance in elite female field hockey players. Br. J. Sports Med., 39(11): 860-865. DOI: 10.1136/ bjsm.2005.019950.

7. Colson S.S., Petit P.D., Hébreard L., Tessaro J., Pensini M. (2009) Whole body vibration does not enhance muscle activation. Int. J. Sports Med., 30(12): 841-844. DOI: 10.1055/s-0029-1234082.

8. Coombs R., Garbutt G. (2002) Developments in the use of the hamstring/ quadriceps ratio for the assessment of muscle balance. J. Sports Sci. Med., 1(3): 56-62. PMID: 24701125.

9. Cordo P., Gurfinkel V.S., Bevan L., Kerr G.K. (1995) Proprioceptive consequences of tendon vibration during movement. J. Neurophysiol., 74(4): 1675-1688. DOI: 10.1152/jn.1995.74.4.1675.

10. Cormie P., Deane R.S., Triplett T., McBride J.M. (2006) Acute effects of whole-body vibration on muscle activity, strength, and power. J. Strength Cond. Res., 20(2): 257-261. DOI: 10.1519/R-17835.1.

11. Coyle E.F., Costill D.L., Lesmes G.E. (1979) Leg extension power and muscle fiber composition. Med. Sci. Sports, 11(1): 12-15.

12. Delecluse C., Roelants M., Verschueren S. (2003) Strength increase after whole-body vibration compared with resistance training. Med. Sci. Sports Exerc., 35(6): 1033-1041. DOI: 10.1249/01.MSS.0000069752.96438.B0.
13. Delextrat A., Cohen D. (2008) Physiological testing of basketball players: toward a standard evaluation of anaerobic fitness. J. Strength Cond. Res., 22(4): 1066-1072. DOI: 10.1519/JSC.0b013e3181739d9b.

14. Dervišević E., Hadžić V. (2012) Quadriceps and hamstrings strength in team sports: basketball, football and volleyball. Isokinet. Exerc. Sci., 20(4): 293-300. DOI: 10.3233/IES-2012-00483.

15. Escamilla R.F. (2001) Knee biomechanics of the dynamic squat exercise. Med. Sci. Sports Exerc., 33(1): 127-141. DOI: 10.1097/00005768-200101000-00020.

16. Hagbarth K.E., Eklund G. (1966) Motor Effects of Vibratory Stimuli in Man. Almqvist and Wiksell: Stockholm.

17. Hawkey A., Faulkner J. (2019) Does acute whole-body vibration training affect the balance, flexibility and explosive power of an Olympic rhythmic gymnast? J. Sports Sci., 37(S1): 75. DOI: 10.1080/02640414.2019.1671688.

18. Hawkey A., Morrison D. (2017) In-season whole-body vibration training enhances vertical jump performance in professional soccer goalkeepers. Turk. J. Sport Exerc., 19(2): 143-149. DOI: 10.15314/tsed.312001.

19. Hawkey A., Rittweger J., Rubin C. (2016) Vibration exercise: evaluating its efficacy and safety on the musculoskeletal system. Sport Exerc. Scientist, 50: 26-27.

20. Holcomb W.R., Rubley M.D., Lee H.J., Guadagnoli M.A. (2007) Effect of hamstring-emphasized resistance training on hamstring: quadriceps strength ratios. J. Strength Cond. Res., 21(1): 41-47. DOI: 10.1519/R-18795.1.

21. Hootman J.M., Dick R., Agel J. (2007) Epidemiology of collegiate injuries for 15 sports: summary and recommendations for injury prevention initiatives. J. Athl. Train., 42(2): 311-319. PMCID: PMC1941297.

22. International Organization for Standardization (ISO). (1997) ISO. 2631-1. In Organization IS (ed). Mechanical vibration and shock: evaluation of human exposure to whole body vibration. Geneva, Switzerland.

23. Jordan M.J., Norris S.R., Smith D.J., Herzog W. (2005) Vibration training: an overview of the area, training consequences, and future considerations. J. Strength Cond. Res., 19(2): 459-466. DOI: 10.1519/13293.1.

24. Keith S.E., Brammer A.J. (1994) Rock drill handle vibration: measurement and hazard estimation. J. Sound Vib., 174(4): 475-491.

25. Kellis E., Baltzopoulos V. (1998) Muscle activation differences between eccentric and concentric isokinetic exercise. Med. Sci. Sports Exerc., 30(11): 1616-1623. DOI: 10.1097/00005768-199811000-00010.

26. Kilduff L.P., Owen N., Bevan H., Bennett M., Kingsley M.I., Cunningham D. (2008) Influence of recovery time on post-activation potentiation in professional rugby players. J. Sport Sci., 26(8): 795-802. DOI: 10.1080/02640410701784517.

27. Krol P., Piecha M., Słomka K., Sobota G., Polak A., Juras G. (2011) The effect of whole-body vibration 
frequency and amplitude on the myoelectric activity of vastus medialis and vastus lateralis. J. Sports Sci. Med., 10(1): 169-174. PMCID: PMC3737908.

28. Luo J., McNamara B., Moran K. (2005) The use of vibration training to enhance muscle strength and power. Sports Med., 35(1): 23-41. DOI: 10.2165/00007256200535010-00003.

29. Madigan M.L., Pidcoe P.E. (2003) Changes in landing biomechanics during a fatiguing landing activity. J. Electromyogr. Kines., 13(5): 491-498. DOI: 10.1016/s10506411(03)00037-3.

30. Mahieu N.N., Witvrouw E., Van de Voorde D., Michilsens D., Arbyn V., Van den Broecke W. (2006) Improving strength and postural control in young skiers: whole-body vibration versus equivalent resistance training. J. Athl. Train., 41(3): 286-293. PMCID: PMC1569559.

31. McLester J.R., Bishop P.A., Smith J., Wyers L., Dale B., Kozusko J., Richardson M., Nerett M.E., Lomax R. (2003) A series of studies - A practical protocol for testing muscular endurance recovery. J. Strength Cond. Res., 17(2): 259-273. DOI: 10.1519/1533-4287(2003)017<0259:asospp>2.0.co;2.

32. Mester J., Spitzenfeil P., Schwarzer J., Seifriz F. (1999) Biological reaction to vibration-implications for sport. J. Sci. Med. Sport, 2(3): 211-226. DOI: 10.1016/s14402440(99)80174-1.

33. Nagura T., Dyrby C.O., Alexander E.J., Andriacchi T.P. (2002) Mechanical loads at the knee joint during deep flexion. J. Orthop. Res., 20(4): 881-886. DOI: 10.1016/ S0736-0266(01)00178-4.

34. Ohkoshi Y., Yasuda K., Kaneda K., Wada T., Yamanaka, M. (1991) Biomechanical analysis of rehabilitation in the standing position. Am. J. Sports Med., 19(6): 605-611. DOI: $10.1177 / 036354659101900609$.

35. Olafsson S., Hawkey A. (2015) Good vibrations: the use of whole body vibration in rehabilitation. Sjukrapjalfarinn, 43(2): 22-29.

36. Pincivero D.M., Aldworth C., Dickerson T., Petry C., Shultz T. (2000) Quadriceps-hamstring EMG activity during functional, closed kinetic chain exercise to fatigue. Eur. J. Appl. Physiol., 81(6): 504-509. DOI: 10.1007/ s004210050075.

37. Ratamess N.A., Alvar B.A., Evetoch T.K., Housh T.J., Kibler W.B., Kraemer W.J., Triplet N.T. (2009) American College of Sports Medicine position stand. Progression models in resistance training for healthy adults. Med. Sci. Sports Exerc., 41: 687-708. DOI: 10.1249/ MSS.0b013e3181915670.

38. Rittweger J. (2010) Vibration as an exercise modality: how it may work and what its potential may be. Eur. J. Appl. Physiol., 108(5): 877-904. DOI: 10.1007/s00421009-1303-3.

39. Rittweger J., Mutschelknauss M., Felsenberg D. (2003) Acute changes in neuromuscular excitability after exhaustive whole body vibration exercise as compared to exhaustion by squatting exercise. Clin. Physiol. Funct. Imaging, 23(2): 81-86. DOI: 10.1046/j.1475-097x.2003.00473.x.

40. Rønnestad B.R., Holden G., Samnøy L.E., Paulsen G. (2012) Acute effect of whole-body vibration on power, one-repetition maximum, and muscle activation in power lifters. J. Strength Cond. Res., 26(2): 531-539. DOI: 10.1519/JSC.0b013e318220d9bb.

41. Siu P.M., Tam B.T., Chow D.H., Guo J.Y., Huang Y.P., Zheng Y.P., Wong S.H. (2010) Immediate effects of 2 different whole-body vibration frequencies on muscle peak torque and stiffness. Arch. Phys. Med. Rehab., 91(10): 1608-1615. DOI: 10.1016/j.apmr.2010.07.214.

42. Teo W., Newton M.J., McGuigan M.R. (2011) Circadian rhythms in exercise performance: implications for hormonal and muscular adaptation. J. Sports Sci. Med., 10(4): 600-606. PMID: 24149547.

43. Weir J.P., Keefe D.A., Eaton J.F., Augustine R.T., Tobin D.M. (1998) Effect of fatigue on hamstring coactivation during isokinetic knee extensions. Eur. J. Appl. Physiol. Occup. Physiol., 78(6): 555-559. DOI: 10.1007/ s004210050460.

44. Westerblad H., Allen D.G., Bruton J.D., Andrade F.H., Lännergren J. (1998) Mechanisms underlying the reduction of isometric force in skeletal muscle fatigue. Acta Physiologica Scandinavica, 162(3): 253-260. DOI: 10.1046/j.1365-201X.1998.0301f.x.

45. Westing S.H., Cresswell A.G., Thorstensson A. (1991) Muscle activation during maximal voluntary eccentric and concentric knee extension. Eur. J. Appl. Physiol. Occup. Physiol., 62(2): 104-108. DOI: 10.1007/bf00626764.

46. World Medical Association. (2013) World Medical Association Declaration of Helsinki: ethical principles for medical research involving human subjects. Journal of the American Medical Association, 310: 2191-2194. DOI: 10.1001/jama.2013.281053.

47. Wright G.A., Delong T.H., Gehlsen G. (1999) Electromyographic activity of the hamstrings during performance of the leg curl, stiff-leg deadlift, and back squat movements. J. Strength Cond. Res., 13(2): 168-174. ISSN Print: 1064-8011.

48. Wright J., Ball N., Wood L. (2009) Fatigue, h/q ratios and muscle coactivation in recreational football players. Isokinet. Exerc. Sci., 17(3): 161-167. DOI: 10.3233/IES2009-0348

\section{Received 17.01.2020 \\ Accepted 18.05.2020}

(C) University of Physical Education, Warsaw, Poland 